POS $\quad$ PROCEEDINGS

\title{
Performance of large area PMTs at cryogenic temperatures for neutrino and rare event physics experiments
}

\author{
Andrea Falcone* \\ Universita' di Pavia - INFN Pavia \\ E-mail: andrea.falconeapv.infn.it
}

\section{Fabrizio Boffelli}

Universita' di Pavia - INFN Pavia

E-mail: fabrizio.boffelli@pv.infn.it

\section{Maurizio Bonesini}

INFN Milano Bicocca

E-mail: maurizio.bonesini@mib.infn.it

\section{Tommaso Cervi}

Universita' di Pavia

E-mail: tommaso.cervi@pv.infn.it

\section{Roberto Mazza}

INFN Milano Bicocca

E-mail: roberto.mazza@mib.infn.it

\author{
Alessandro Menegolli \\ Universita' di Pavia - INFN Pavia \\ E-mail: alessandro.menegolli@pv.infn.it
}

Claudio Montanari

INFN Pavia

E-mail: claudio.montanari@pv.infn.it

Marco Prata

INFN Pavia

E-mail: marco.prata@pv.infn.it

Andrea Rappoldi

INFN Pavia

E-mail: andrea.rappoldi@pv.infn.it

Gian Luca Raselli

INFN Pavia

E-mail: gianluca.raselliepv.infn.it

Massimo Rossella 
INFN Pavia

E-mail: massimo.rossella@pv.infn.it

Maura Spanu

Universita' di Pavia - INFN Pavia

E-mail: maura.spanu@pv.infn.it

\section{Marta Torti}

Universita' di Pavia - INFN Pavia

E-mail: marta.torti@pv.infn.it

\section{Andrea Zani}

INFN Pavia

E-mail: andrea.zani@pv.infn.it

An evaluation of the behavior of three large cathode area photo-multiplier tubes, Hamamatsu R5912 Mod and R5912-02 Mod, and ETL 9357 KFLB, was carried out both at room temperature and immersed in liquid nitrogen, at a temperature of $77 \mathrm{~K}$. The main electrical and optical features of the devices were studied: signal shape, photo-cathode response uniformity, gain, linearity and dark count rate. An evaluation of the quantum efficiency was also made in the vacuum ultraviolet light region.

International Conference on New Photo-detectors, PhotoDet2015

6-9 July 2015

Moscow, Troitsk,Russia

${ }^{*}$ Speaker. 


\section{Introduction}

Measurement of scintillation light in liquefied noble gases plays a very important role in many detectors dedicated to neutrino physics and Dark Matter search. Photo Multipliers Tubes (PMTs) represent presently the preferred readout devices to collect light in large volume detectors, as those needed for rare events physics. Various experiments already used large area (8 inches) PMTs directly immersed in liquid Ar or Xe at cryogenic temperature. In view of future applications, three new large area PMTs, Hamamatsu R5912 Mod and R5912-02 Mod and ETL 9357 KFLB (see also [1,2]), were characterized both at room (see Section 2 and 3) and at cryogenic temperature (see Section 4). The work presented in $[3,4]$ was completed by studying the poor linearity response of Hamamatsu R5912-02 PMTs and by characterizing the ETL 9357 KFLB PMT at cryogenic temperature, using a new PMT specimen, with no problem on photo-cathode uniformity response.

\section{Characterization at room temperature}

PMTs under analysis have a 8-inch diameter sand-blasted window made of borosilicate glass and a bialkali photo-cathode $\left(\mathrm{K}_{2} \mathrm{CsSb}\right)$ with platinum undercoating, in order to restore the photo-cathode conductivity at low temperature. Hamamatsu R5912 Mod and R5912-02 Mod PMTs have 10 and 14 dynodes, respectively, while the ETL 9357 KFLB has 12 stages.

PMTs were illuminated with a $405 \mathrm{~nm}$ laser diode ${ }^{1}$, using a pulse generator ${ }^{2}$ and an optical fiber $^{3}$. A proper support was used to maintain the fiber at a fixed orientation, normal to the PMT window, while allowing to move it in various positions on the window itself. A charge sensitive preamplifier ${ }^{4}$ and a shaping amplifier ${ }^{5}$ were used to form the PMT signals, then acquired with a Multi Channel Analyzer ${ }^{6}$.

Single photo electron response (SER) was studied, as a function of the position of the fiber along two perpendicular diameters, to estimate photo-cathode uniformity. Hamamatsu PMTs showed a good uniformity, within $10 \%$, up to $10 \mathrm{~cm}$ from the tube axis, where a gain reduction occurs, probably due to the electric field non uniformity in the peripheral region of the tube (see also [3, 4]). This behavior does not occur with ETL 9357 KFLB, where the uniformity remains within $10 \%$ up to the border of the PMT windows (see Fig. 1).

A different experimental setup was used to measure the quantum efficiency (Q.E.) of the photocathodes in the vacuum ultraviolet (VUV) light region. The PMT under test was placed inside a vacuum chamber optically connected to a VUV monochromator ${ }^{7}$. In order to be sensitive to VUV light, the PMT windows were coated with Tetra-Phenyl-Butadiene (TPB), a wavelength-shifter, with emission peak at $430 \mathrm{~nm}$. The experimental setup includes a scanner ${ }^{8}$, a Deuterium lamp ${ }^{9}$, a

\footnotetext{
${ }^{1}$ NICHIA NDV1413 LASER diode

${ }^{2}$ Avtech AVO-9A-C-P2-LARB

${ }^{3} 7 \mu \mathrm{m}$ core diameter, $3 \mathrm{~m}$ long

${ }^{4}$ CANBERRA 2005

${ }^{5}$ ORTEC-570

${ }^{6}$ ORTEC-Easy-8k, 12 bit

${ }^{7}$ model McPHERSON 234/302

8 model McPHERSON 789A-3

${ }^{9}$ model McPHERSON 632
} 

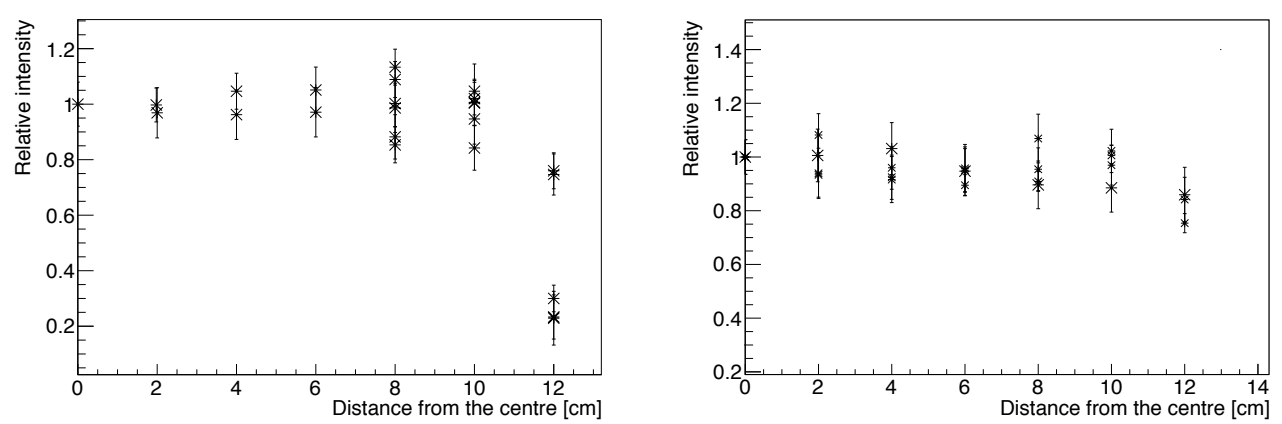

Figure 1: Relative response as a function of the distance from the center of the window, along different diameters, for Hamamatsu R5912 (left) and ETL 9357 KFLB (right). Different measurements were made for each position and error bars represent the statistical errors.

rotating $\mathrm{Al}+\mathrm{MgF}_{2}$ mirror, a NIST calibrated reference photo-diode ${ }^{10}$ and collimating optics. The whole system was set under vacuum conditions, down to $10^{-4}$ mbar, to prevent ultraviolet light absorption. Thanks to the rotating mirror, the light spot was directed alternatively on the PMT surface or on the reference photo-diode. A wavelength-dependent analysis was performed from $120 \mathrm{~nm}$ to $220 \mathrm{~nm}$ (see Fig. 2). The Q.E. was obtained by comparing the current measured with the PMT to the one collected with the reference diode, keeping the illumination constant. Measurements were carried out by means of a picoammeter ${ }^{11}$. For LAr (LXe) emission peak, i.e. for $\lambda=128 \mathrm{~nm}$ $(\lambda=178 \mathrm{~nm}), \mathrm{R} 5912$ and $9357 \mathrm{KFLB}$ show a Q.E. of $7.0 \% \pm 0.6 \%(6.0 \% \pm 0.5 \%)$ and $4.7 \% \pm 0.7 \%$ $(4.4 \% \pm 0.6 \%)$ respectively.
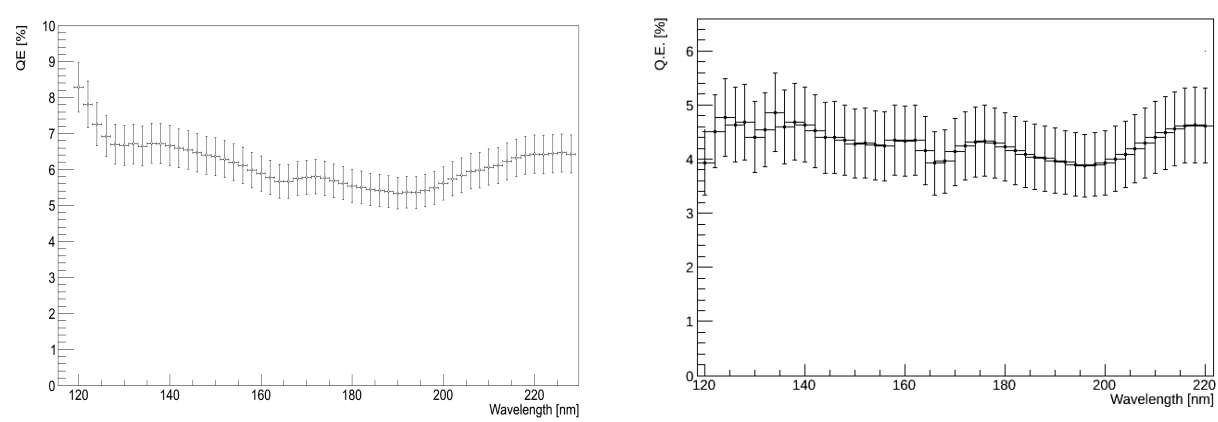

Figure 2: Quantum efficiency for $120 \div 220 \mathrm{~nm}$ incident light of Hamamatsu R5912 (left) and ETL 9357 KFLB (right).

\section{Characterization at cryogenic temperature}

The PMTs were directly immersed in liquid nitrogen $(\mathrm{T}=77 \mathrm{~K})$, to test them in real experimental conditions. Measurements were carried out after $\sim 3$ days of rest in the cryogenic environment,

\footnotetext{
${ }^{10}$ model AXUV-100

${ }^{11}$ model Keithley $6487 \mathrm{E}$
} 
that is the time needed for the stabilization of the PMT characteristics. Signal shape and transit time measurements, at SER condition and cryogenic temperature, are reported in Table 1.

\begin{tabular}{cccc}
\hline & R5912 & R5912-02 & 9357 KFLB \\
\hline \hline Leading edge (ns) & $3.8 \pm 1.1$ & $3.6 \pm 0.9$ & $4.0 \pm 2.0$ \\
FWHM (ns) & $4.4 \pm 0.1$ & $5.0 \pm 0.8$ & $6.4 \pm 3.2$ \\
Transit time (ns) & $55.1 \pm 1.5$ & $70.1 \pm 1.5$ & $66.4 \pm 2.2$ \\
\hline
\end{tabular}

Table 1: PMTs characteristics, at SER condition and cryogenic temperature, of tested devices.

For the test at cryogenic temperature, the same set-up and acquisition system described in Section 2 were used, with the fiber and the other cables allowed to enter by a proper feed-through, used to preserve darkness conditions and thermal insulation (see [3, 4]). The gain of the devices was estimated from SER fit curves as a function of the applied voltage and temperature. A gain reduction occurring at $77 \mathrm{~K}$ was evident, as expected, for both Hamamatsu and ETL devices, being $\sim 70 \%$ in the R5912 and 9357 KFLB and 35\% in the R5912-02 (see Fig. 3).

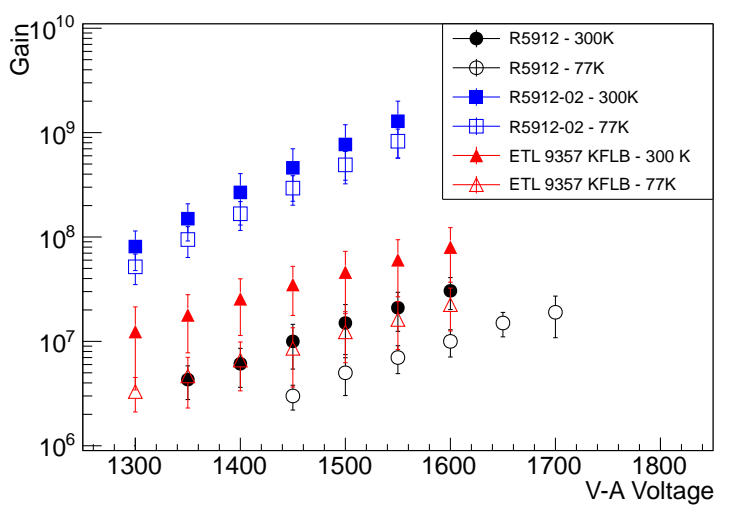

Figure 3: Gain trends for tested PMTs at room (full markers) and at cryogenic (empty markers) temperature as a function of the voltage between anode and cathode.

The linearity of the devices was studied by illuminating them with increasing light intensity. To perform this, a series of neutral density optical filters, calibrated with the same light used in the measurements and mounted on two rotating supports, were used ${ }^{12}$. Starting from the maximum attenuation it was possible to increase the illumination intensity and to study the linearity of the devices. Measurements were carried out with a pulsed light source (2 ns width) at a repetition rate of $20 \mathrm{~Hz}$. Both the peak amplitude and the collected charge distribution were studied. The ETL 9357 KFLB PMT reached saturation after $\sim 200$ phe. R5912 remained linear up to 400 phe [3, 4], while R5912-02 reached the saturation regime after a few phe, $\sim 10$. Important differences were not detected between room and cryogenic temperature (see Fig. 4).

To better understand the linearity losses of R5912-02 additional dedicated tests were carried out, at room temperature. The linearity of the anode signal resulted to depend on the gain: as shown

\footnotetext{
${ }^{12}$ Attenuation factors of the filters (for $405 \mathrm{~nm}$ light) are $11.0 \pm 0.1,31.9 \pm 0.3,109.9 \pm 1.0$ and $3.36 \pm 0.04,1.76 \pm 0.02$, $2.85 \pm 0.30,6.52 \pm 0.06,5515 \pm 50$ for the two rotating support respectively.
} 

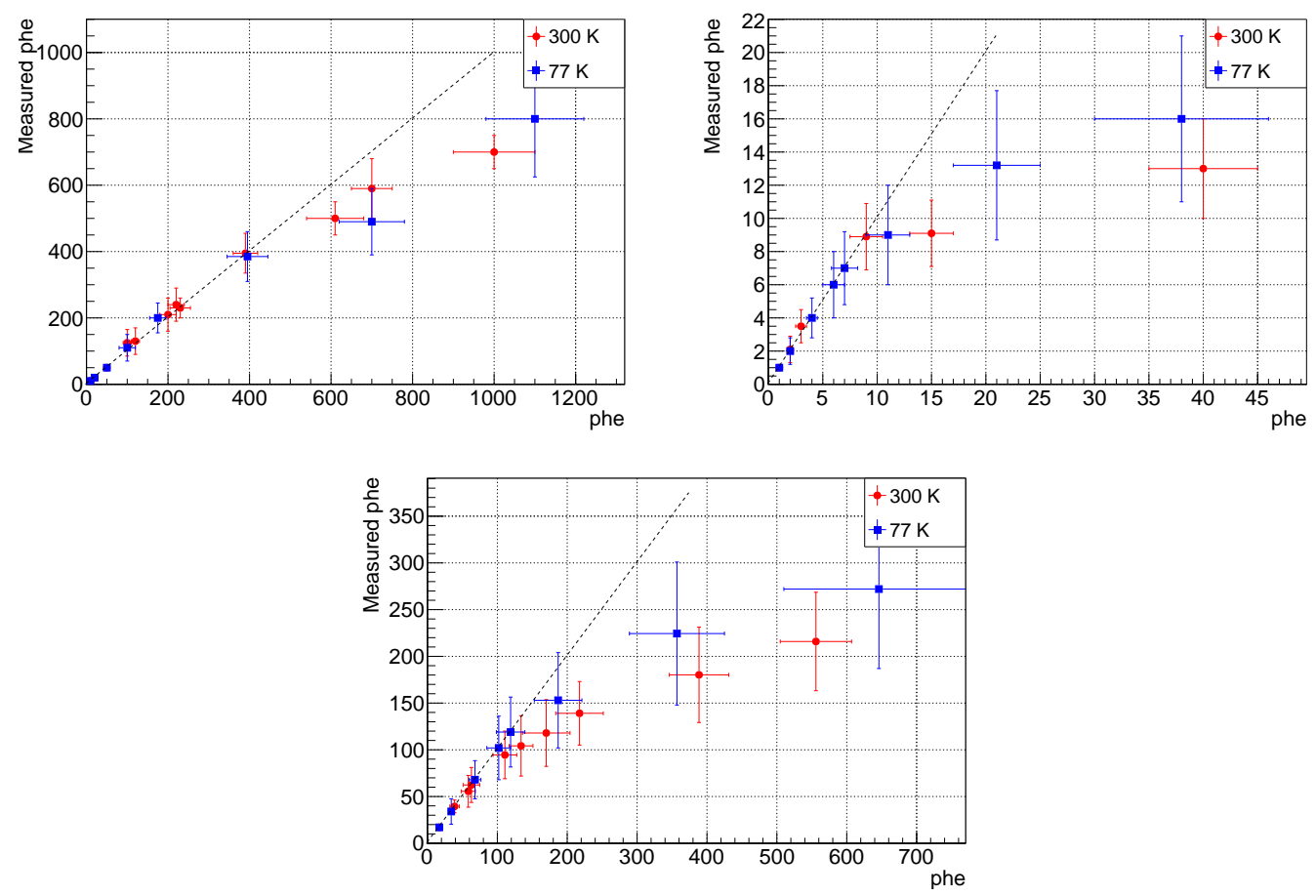

Figure 4: Linearity of R5912 (top-left), R5912-02 (top-right) and 9357 KFLB (bottom) at room and at cryogenic temperature.

in Fig. 5, the PMT reaches saturation earlier at higher gain. It seemed to be independent from the amount of current drained by the bases: no difference in linearity behavior was noticed changing the values of resistors in the PMT basis. In order to understand where, along the PMT multiplication stage, the amplification problems arise, the signal from the last dynodes (down to $8^{\text {th }}$ ) was picked up. Saturation effects were still present in the dynodic signals, and the behavior reproduced the anodic non-linearity (see Fig. 5). Saturation problems, connected to the PMT gain, suggested induced effects by the dynodic chain, but not localized in the last part of the amplification stage: they could be connected to the internal design of the amplification stage.

The PMTs dark count rate was measured with a different acquisition system, i.e. with a discriminator ${ }^{13}$ and a counter ${ }^{14}$. The discrimination threshold was gradually increased from 1 to $255 \mathrm{mV}$, with $1 \mathrm{mV}$ steps. Results are presented in Fig. $6[3,4]$. The spectrum structure of Hamamatsu devices is characterized by a clear bump profile centered around one phe, caused mainly by the cathode dark noise. This bump is not present in ETL 9357 KFLB, where no single electron signal was detectable when studying voltage peak, due to the great instability of the signal shape: this compromised the possibility to set good threshold for the dark noise measurements. The dark count rate increased with decreasing temperature: this effect is referred as Non-Thermal Dark Rate. The source of this emission is found in the photo-cathode, but its nature and features are not yet understood [6]. It could be ascribed to a decrease of the lattice-energy of the cathode material

\footnotetext{
${ }^{13}$ CAEN V812

${ }^{14}$ CAEN V560
} 

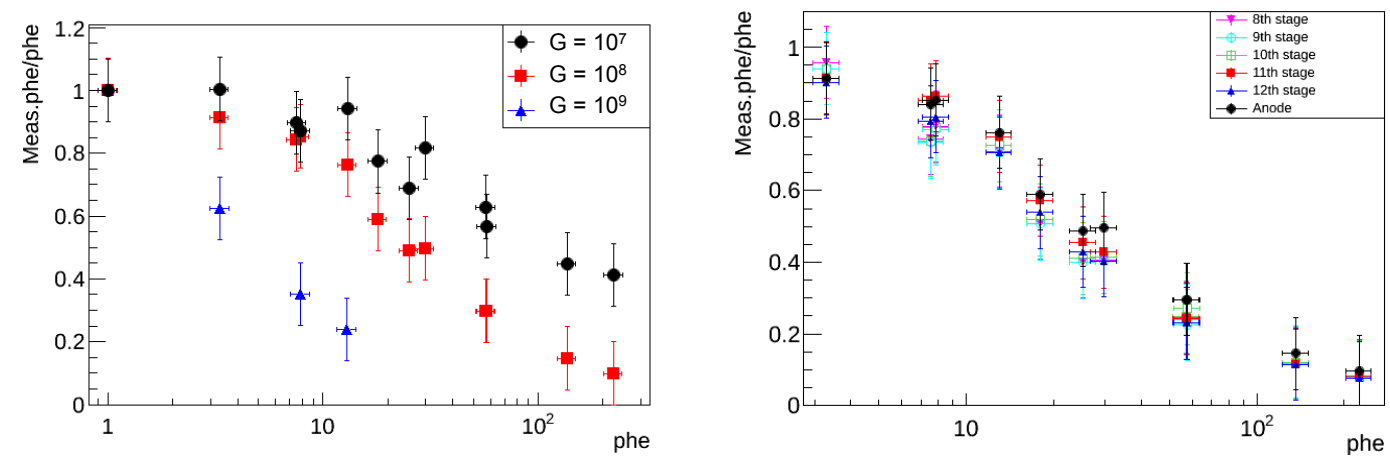

Figure 5: Left: Linearity response of R5912-02 for three different gains. Right: Linearity response of Hamamatsu R5912-02 for a gain of $10^{8}$. Signals are collected both on the anode and on the last multiplication electrodes (down to the $8^{\text {th }}$ dynode). In both graphs the signal (Meas.phe) is normalized to the expected phe numbers for the ideal case (phe).

at low temperature, resulting in an increase of the electron escape probability [5]. R5912-02, at room temperature, showed a dark count rate lower than $0.4 \mathrm{kHz}$ for all thresholds; the increase at $\mathrm{T}=77 \mathrm{~K}$ is very pronounced, but the rate remains lower than $2 \mathrm{kHz}$. For R5912 dark count rate is higher, both at room $(\geq 0.4 \mathrm{kHz}$ till 0.9 phe of threshold $[3,4])$ and cryogenic ( $\geq 2 \mathrm{kHz}$ till 0.6 phe of threshold [3,4]) temperature. 9357 KFLB present lower dark counts and also lower increase at cryogenic temperature, being the rate $\leq 1 \mathrm{kHz}$ for almost all the threshold values also at $\mathrm{T}=77 \mathrm{~K}$.

\section{Conclusion}

Three different 8“ large area photo-multipliers, Hamamatsu R5912 and R5912-02 and ETL 9357 $\mathrm{KFLB}$, to be used in future liquefied noble gas detectors, were characterized both at room and cryogenic temperature. This work complements the analysis presented in $[3,4]$. All tested tubes showed a good photo-cathode uniformity and Q.E. value for VUV light. The tested photon detectors showed a good behavior and are suitable for cryogenic application:

- the gain decrease at low temperature was $\sim 70 \%$ in the R5912 and 9357 KFLB PMTs, and $\sim 35 \%$ in the R5912-02 PMT;

- linearity up to 400 and 200 phe was achieved for the R5912 and ETL 9357 KFLB PMTs respectively, while saturation occurred after $\sim 10 \%$ phe for the R5912-02 PMT;

- the saturation problems of R5912-02 PMT did not seem to be related to the dynode biasing, but connected to the internal design of the amplification stage;

- a relatively high dark counts rate was found for Hamamatsu devices, while ETL PMT presented a lower noise. 

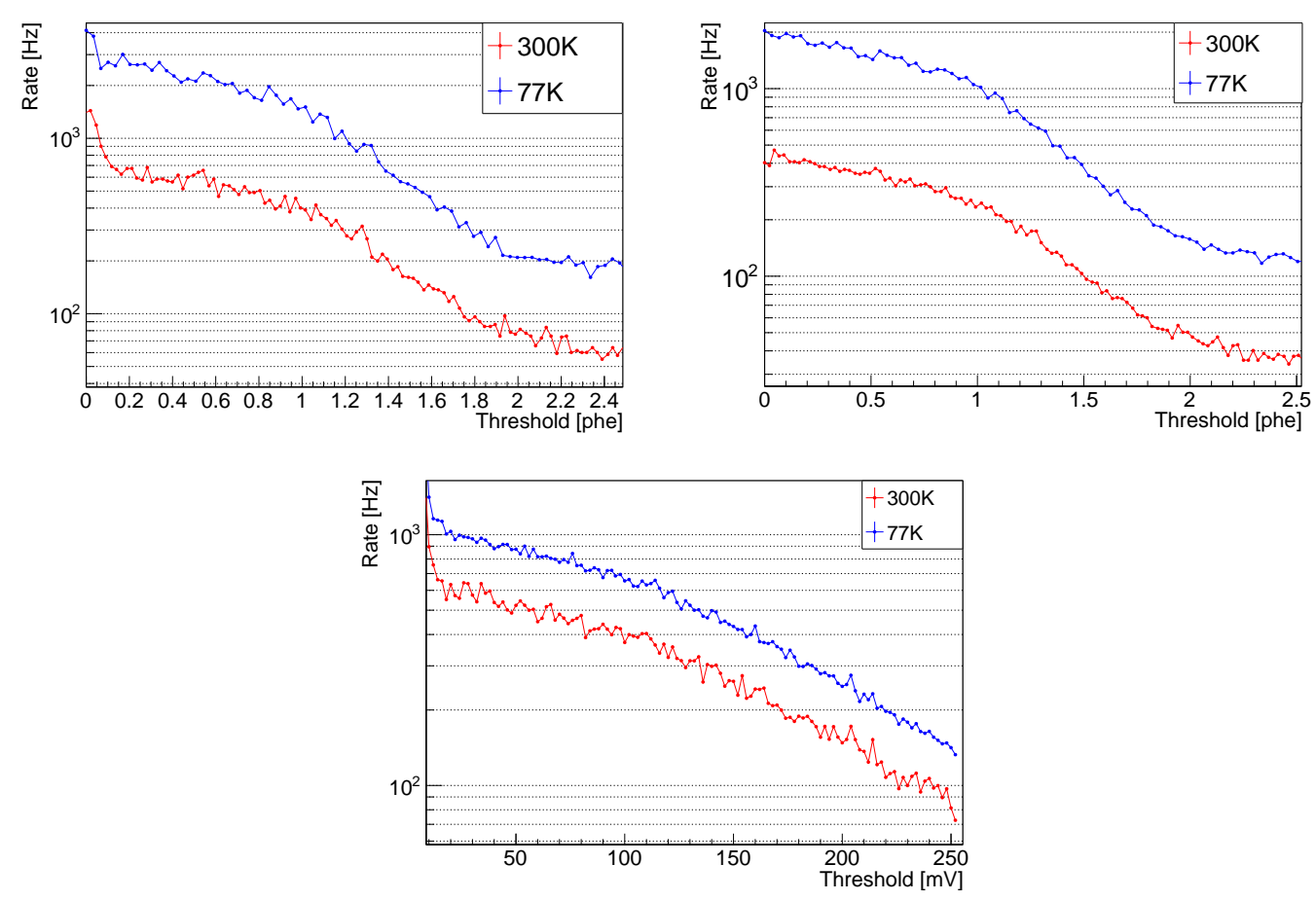

Figure 6: Dark count spectra for R5912 (top-left), R5912-02 (top-right) and ETL 9357 KFLB (bottom) at room and at cryogenic temperature.

\section{References}

[1] A.Bueno et al., Characterization of large area photomultipliers and its application to dark matter search with noble liquid detectors, JINST 3 (2008) P01006.

[2] T.Briese et al., Testing of cryogenic photomultiplier tubes for the MicroBooNE experiment, JINST 8 (2013) T07005.

[3] A.Falcone et al., Comparison between large area photo-multiplier tubes at cryogenic temperature for neutrino and rare event phisics experiments, in proceedings of NDIP 2014, Nucl. Instrum. and Meth. A 787 (2015) 55-58.

[4] P.Agnes et al., Characterization of large area PMTs at cryogenic temperature for rare event physics experiments, JINST 9 (2014) C03009.

[5] A.Ankowski et al., Characterization of ETL 9357FLA photomultiplier tubes for cryogenic temperature applications, Nucl. Instrum. and Meth. A 556 (2006) 146-157.

[6] H.O.Mayer, Dark rate of a photomultiplier at cryogenic temperatures, [arXiv: $0805.0771 \mathrm{v} 1$ (nucl-ex)]. 